\title{
A new genomic prediction method with additive-dominance effects in the least-squares framework
}

\author{
Hailan Liu $\mathbb{1}^{1}{ }^{1}$ Guo-Bo Chen $\mathbb{1}^{2}$
}

Received: 31 January 2018 / Accepted: 23 May 2018 / Published online: 20 June 2018

(c) The Genetics Society 2018

\begin{abstract}
In our previous work, we proposed a genomic prediction method combing identical-by-state-based Haseman-Elston regression and best linear prediction with additive variance component only (HEBLPIA herein), the most essential component of genetic variation. Since the dominance effects contribute significantly in heterosis, it is desirable to incorporate the HEBLP with dominance variance component that is expected to enhance the predictive accuracy as we move to the further development: HEBLPIAD, a paralleled implementation of genomic prediction compared with genomic best linear unbiased prediction (GBLUP). The simulation results indicated that when the dominance effects contributed to a large proportion of genetic variation, HEBLP|AD and GBLUPIAD, having similar accuracy, both outperformed HEBLP|A; but when the dominance variation was none or little, HEBLPIA, HEBLPIAD, and GBLUPIAD had similar predictability. The analysis of real data from Arabidopsis thaliana F2 population also demonstrated the latter situation. In summary, HEBLPI $\mathrm{AD}$ performed stable whether a trait was controlled by dominance effects or not.
\end{abstract}

\section{Introduction}

With the rapid development of high-throughput molecular marker techniques, such as single nucleotide polymorphisms (SNPs) and statistical approaches, genomic prediction first proposed by Meuwissen et al. (2001) has been successfully applied to genetic improvement of complex traits that are controlled by polygenic effects-numerous smalleffect quantitative trait loci (QTL) (Schaeffer, 2006; Hayes et al. 2009; Jannink et al. 2010; Zhang et al. 2011; Riedelsheimer et al. 2012). Compared to the conventional marker-assisted selection (MAS), genomic prediction is far more accurate by utilizing all molecular marker information to estimate the breeding values of each individual in a

Hailan Liu

lhlzju@ hotmail.com

$\triangle$ Guo-Bo Chen

chenguobo@gmail.com

1 Maize Research Institute, Sichuan Agricultural University, Chengdu, Sichuan Province 611130, China

2 Clinical Research Institute, Zhejiang Provincial People's Hospital, People's Hospital of Hangzhou Medical College,

Hangzhou 310014 Zhejiang Province, China candidate population (Heffner et al. 2009; Arruda et al. 2016).

In the early stage of genomic prediction methods, many models accounted only for additive effects (Meuwissen et al. 2001; Bernardo and Yu, 2007; Calus et al. 2008; VanRaden, 2008). However, dominance effects contribute to heterosis (Hua et al. 2003; Li et al. 2008), and therefore should be included in the models orienting hybrid breeding. Recent studies also show that genomic prediction models including dominance effects can improve the prediction accuracy (Denis and Bouvet, 2011; Su et al. 2012; Technow et al. 2012; Denis and Bouvet, 2013; Nishio and Satoh, 2014; de Almeida Filho et al. 2016; Wang et al. 2017; Liu et al. 2017; Resende et al. 2017).

In our previous study, we developed a fast genomic prediction approach (namely HEBLP, or HEBLPIA herein) combining identical-by-state (IBS)-based Haseman-Elston (HE) regression and best linear prediction (BLP). It can obtain the total additive genetic variance via a simple HE linear regression with reduced computation complexity, but only additive effects are included (Liu and Chen, 2017). The present study aims to develop the HEBLP with both the additive and dominance effects (HEBLPIAD) and to evaluate its predictive performance in the simulated and a real Arabidopsis thaliana $\mathrm{F} 2$ population. 


\section{Materials and methods}

\section{The Arabidopsis thaliana F2 population}

We used the phenotype and genotype data of an Arabidopsis thaliana $\mathrm{F} 2$ population (namely $\mathrm{P} 19)$ derived from a cross between Bay-0 and Lov-5 (Salomé et al. 2011). It consists of 384 individuals and 245 SNP markers. There are seven traits including days until visible flower buds in the center of the rosette (DTF1), days until inflorescence stem reached $1 \mathrm{~cm}$ in height (DTF2), days until first open flower (DTF3), rosette leaf number (RLN), cauline leaf number (CLN), total leaf number: sum of RLN and CLN (TLN), and leaf initiation rate (RLN/DTF1) (LIR1). For more details about the P19 population please refer to Salomé et al. 2011.

\section{Statistical models}

The linear model of a quantitative trait can be written as:

$y=Z_{a} a+Z_{d} d+e$,

in which $y$ is the $n \times 1$ vector for the standardized phenotypic value of a quantitative trait measured from $n$ individuals $\left(y_{i}=\frac{y_{i}^{\prime}-\bar{y}}{\sigma_{y}}\right), y_{i}^{\prime}$ represents the raw phenotypic value; $\bar{y}$ represents the mean value of the phenotypic values; and $\sigma_{y}$ represents the standard error of the phenotypic values.); $Z_{a}$ is the standardized genotype matrix of $n$ rows and $m$ columns for additive effects ( $m$ represents the number of markers.). $Z_{d}$ is the standardized genotype matrix $n \times m$ for dominance effects. In order to keep the additive and dominance variances orthogonal to each other, the coding schemes for additive and dominance effects should be tuned accordingly (Vitezica et al. 2017). For the $i$ th individual at the $k$ th locus, $Z_{a, i k}=\frac{x_{i k}-2 p_{k}}{\sqrt{2 p_{k}\left(1-p_{k}\right)}}$, in which $x_{i k}$ counts the number of reference alleles $(2,1$, and 0 for $\mathrm{AA}, \mathrm{Aa}$, and aa, respectively) and $p_{k}$ the frequency of the reference allele $\mathrm{A}$ at the locus. $Z_{d, i k}=\frac{\delta_{i k}-2 p k}{2 p k(1-p k)}$, in which $\delta_{i k}$ is coded $0,2 p_{k}$, and $\left(4 p_{k}-2\right)$, respectively for $A A, A a$, and $a a$ genotypes, respectively. F2 population the expected $p k$ is 0.5 , and the frequency for $A A, A a$, and $a a$ are $0.25,0.5$, and 0.25 , respectively, under the Hardy-Weinberg equilibrium. The additive and dominance effects of the causal loci were represented by $a$ and $d$, respectively; the additive effects follow $N\left(0, \sigma_{d}^{2}\right)$; the dominance effects follow $N\left(0, \sigma_{d}^{2}\right)$; and $e$ is the residual error, following $N\left(0, \sigma_{e}^{2}\right)$. Therefore, $\operatorname{var}(y)=\Omega_{a} \sigma_{a}^{2}+\Omega_{d} \sigma_{d}^{2}+I \sigma_{e}^{2}$, in which $\Omega_{a}=\frac{z_{a} z_{a}^{\prime}}{m}$ is the additive genetic relationship matrix and $\Omega_{d}=\frac{z_{d} z_{d}}{m}$ is the dominance genetic relationship matrix.
For HEBLPIA and HEBLPIAD methods, we estimated total additive $\left(\sigma_{a}^{2}\right)$ and dominance $\left(\sigma_{d}^{2}\right)$ genetic variance in the training population via Haseman-Elston regression (HE) as below

$Y=b_{0}+b_{a} \omega_{a}+b_{d} \omega_{d}+\varepsilon$,

in which $Y$ is a vector of $\frac{n(n-1)}{2}$ elements for the squared difference between a pair of individuals and $Y_{i j}=\left(y_{i}-y_{j}\right)^{2}$; $\omega_{a}$ is the additive genetic relatedness between a pair of individuals $i$ and $j$, as found in the $i$ th row and the $j$ th column entry in $\Omega_{a} ; \omega_{d}$ is the dominance genetic relatedness between a pair of individuals $i$ and $j$, similarly as found in the $i$ th row and the $j$ th column entry in $\Omega_{d}$. Alternative to HE, linear mixed model can be employed to estimate the additive and dominance variance components via restricted maximum likelihood (REML) algorithm. Of note, the difference between HE and linear mixed model are as below. HE is based on least squares, and it allows the analytical result for $b_{a}$ and $b_{d}$, respectively. In contrast, REML is a model-based approach and the exact structure of the estimated variance, regardless of additive or dominance, remains elusive. Furthermore, as discussed in our previous study (Liu and Chen, 2017), the computational complex for HE is $\mathcal{O}\left(n^{2}\right)$, proportional to the square of sample size, but for REML $\mathcal{O}\left(n^{3}\right)$. The computational advantage of $\mathrm{HE}$ is important especially when the sample size is large.

\section{Analytical results for the Haseman-Elston regression}

The least-squares framework exists analytical results for the regression coefficient. Although, Eq 2 is a linear model of two regression coefficients, $E\left(b_{a}\right)=\frac{\operatorname{cov}\left(Y, \omega_{a}\right)}{\operatorname{var}\left(\omega_{a}\right)}$ and $E\left(b_{d}\right)=$ $\frac{\operatorname{cov}\left(Y, \omega_{d}\right)}{\operatorname{var}\left(\omega_{d}\right)}$ because $\omega_{a}$ and $\omega_{d}$ are orthogonal for each locus. The general principal for deriving the analytical solution for $E\left(b_{a}\right)$ can be found in Chen's study (Chen, 2014). For $E\left(b_{a}\right)$, $\operatorname{cov}\left(Y, \omega_{a}\right)=E\left(Y \omega_{a}\right)-E(Y) E\left(\omega_{a}\right)=E\left(Y \omega_{a}\right)$ because $E$ $(Y)=0$.

$$
E\left(Y \omega_{a}\right)=\frac{1}{m} \sum_{x_{i k}} \sum_{x_{j k}} \omega_{a, i k} \omega_{a, j k}\left[E\left(y_{i} \mid x_{i k}\right)-E\left(y_{j} \mid x_{j k}\right)\right]^{2} p\left(x_{i k}\right) p\left(x_{j k}\right),
$$

in which $E\left(y_{i} \mid x_{i k}\right)$ is the conditional probability of the phenotype given its genotype, $\omega_{a, i k}$ as defined above. $p\left(x_{i k}\right)$ takes value of $0.25,0.5$, and 0.25 , respectively, given $x_{i k}=$ $A A, A a$, and $a a$. In quadric form

$E\left(Y \omega_{a}\right)=\frac{1}{m} \boldsymbol{\beta}^{T} \boldsymbol{I}_{\boldsymbol{A}}\left\{\sum_{k=1}^{m} \mathcal{M}_{k}\right\} \boldsymbol{I}_{\boldsymbol{A}} \boldsymbol{\beta}$,

in which the general form of $\boldsymbol{\beta}^{T}=\left[\beta_{1}+\left(p_{1}-q_{1}\right) d_{1}, \beta_{2}+\right.$ $\left.\left(p_{2}-q_{2}\right) d_{2}, \ldots, \beta_{m}+\left(p_{m}-q_{m}\right) d_{m}\right]$ the vector for additive 
effects and $\boldsymbol{I}_{A}$ an identity matrix with $\boldsymbol{I}_{A, k k}=\sqrt{2 p_{k} q_{k}}$. For F2 populations, as ${ }_{p i}=0.5$ the dominance effect $d_{i}$ will be eliminated out from $\boldsymbol{\beta}$.

$\mathcal{M}_{k}=\left(\begin{array}{cccc}\rho_{1, k}^{2} & \rho_{2, k} \rho_{1, k} & \cdots & \rho_{m, k} \rho_{1, k} \\ \rho_{1, k} \rho_{2, k} & \rho_{2, k}^{2} & \cdots & \rho_{m, k} \rho_{2, k} \\ \vdots & \vdots & \ddots & \vdots \\ \rho_{1, k} \rho_{m, k} & \rho_{2, k} \rho_{m, k} & \cdots & \rho_{m, k}^{2}\end{array}\right)$,

a symmetric matrix, indicating how the $k$ th marker tags QTLs; for instance the entry at the $i$ th row and the $j$ th column $\rho_{i, k}, \rho_{j, k}$ represents the joint LD of the $i$ th and the $j$ th QTLs tagged by the $k$ th marker.

The denominator $\operatorname{var}\left(\omega_{a}\right)$ can be written as $\frac{1}{m^{2}} \sum_{k_{1}=1}^{m} \sum_{k_{2}=1}^{m} \rho_{k_{1} k_{2}}^{2}$, understood as the averaged linkage disequilibrium between each pair of markers-including a marker with itself (see Appendix for the definition of effective number of markers $\left.m_{e}\right)$. Alternatively, $\operatorname{var}\left(\omega_{a}\right)$ can be expressed in quadric form

$$
\operatorname{var}\left(\omega_{a}\right)=\frac{1}{m^{2}} 1^{T}\left(\begin{array}{cccc}
1 & \rho_{2,1}^{2} & \cdots & \rho_{1, m}^{2} \\
\rho_{1,2}^{2} & 1 & \cdots & \rho_{2, m}^{2} \\
\vdots & \vdots & \ddots & \vdots \\
\rho_{1, m}^{2} & \rho_{2, m}^{2} & \cdots & 1
\end{array}\right) 1
$$

in which $1^{T}=[1,1, \ldots 1]$ a vector for 1 .

So, in quadric form

$$
E\left(b_{a}\right)=-2 m \frac{\boldsymbol{\beta}^{T} \boldsymbol{I}_{\boldsymbol{A}}\left\{\sum_{k=1}^{m}\left(\begin{array}{cccc}
\rho_{1, k}^{2} & \rho_{2, k} \rho_{1, k} & \cdots & \rho_{m, k} \rho_{1, k} \\
\rho_{1, k} \rho_{2, k} & \rho_{2, k}^{2} & \cdots & \rho_{m, k} \rho_{2, k} \\
\vdots & \vdots & \ddots & \vdots \\
\rho_{1, k} \rho_{m, k} & \rho_{2, k} \rho_{m, k} & \cdots & \rho_{m, k}^{2}
\end{array}\right)\right\} \boldsymbol{I}_{\boldsymbol{A}} \boldsymbol{\beta}}{1^{T}\left(\begin{array}{cccc}
1 & \rho_{2,1}^{2} & \cdots & \rho_{1, m}^{2} \\
\rho_{1,2}^{2} & 1 & \cdots & \rho_{2, m}^{2} \\
\vdots & \vdots & \ddots & \vdots \\
\rho_{1, m}^{2} & \rho_{2, m}^{2} & \cdots & 1
\end{array}\right) 1} .
$$

Similarly, for $E\left(b_{d}\right)$, we had

$E\left(Y \omega_{d}\right)=$

$\frac{1}{m} \sum_{x_{i k}} \sum_{x_{j k}} \omega_{d, i k} \omega_{d, j k}\left[E\left(y_{i} \mid x_{i k}\right)-E\left(y_{j} \mid x_{j k}\right)\right]^{2} p\left(x_{i k}\right) p\left(x_{j k}\right)$ and its quadric form

$\frac{1}{m} \boldsymbol{D}^{T} \boldsymbol{I}_{D}\left\{\sum_{k=1}^{m}\left(\begin{array}{cccc}\rho_{1, k}^{4} & \rho_{2, k}^{2} \rho_{1, k}^{2} & \cdots & \rho_{m, k}^{2} \rho_{1, k}^{2} \\ \rho_{1, k}^{2} \rho_{2, k}^{2} & \rho_{2, k}^{4} & \cdots & \rho_{m, k}^{2} \rho_{2, k}^{2} \\ \vdots & \vdots & \ddots & \vdots \\ \rho_{1, k}^{2} \rho_{m, k}^{2} & \rho_{2, k}^{2} \rho_{m, k}^{2} & \cdots & \rho_{m, k}^{4}\end{array}\right)\right\} \boldsymbol{I}_{D} \boldsymbol{D}$

in which $\boldsymbol{D}=\left[d_{1}, d_{2}, \ldots d_{m}\right]$ the vector for dominance effects and $\boldsymbol{I}_{\mathrm{D}}$ an identity matrix with $\boldsymbol{I}_{D, k k}=2 p_{k} q_{k}$. The denominator is $\operatorname{var}\left(\omega_{d}\right)=\frac{1}{m^{2}} \sum_{k_{1}=1}^{m} \sum_{k_{2}=1}^{m} \rho_{k_{1} k_{2}}^{4}$, and in
quadric form

$$
\operatorname{var}\left(\omega_{d}\right)=\frac{1}{m^{2}} 1^{T}\left(\begin{array}{cccc}
1 & \rho_{2,1}^{4} & \cdots & \rho_{1, m}^{4} \\
\rho_{1,2}^{4} & 1 & \cdots & \rho_{2, m}^{4} \\
\vdots & \vdots & \ddots & \vdots \\
\rho_{1, m}^{4} & \rho_{2, k}^{4} & \cdots & 1
\end{array}\right) 1
$$

So,

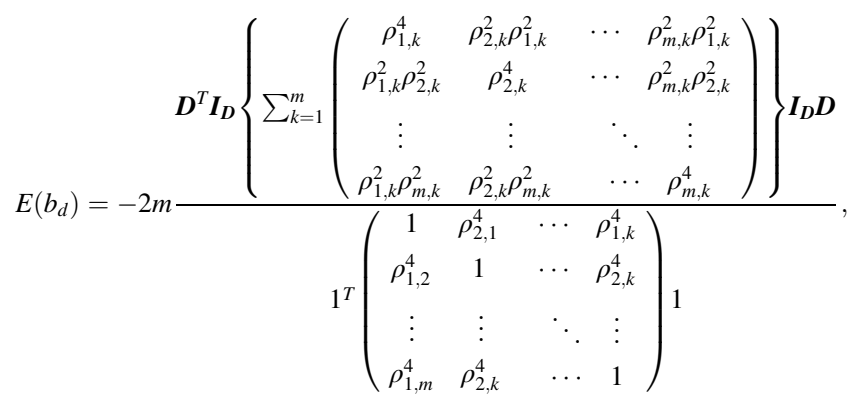

Although, $E\left(b_{a}\right)$ and $E\left(b_{d}\right)$ resemble each other, $E\left(b_{a}\right)$ has its kernel related to squared correlation $\rho^{2}$, which is a term associated to the additive variance (Hill and Robertson, 1968), while $E\left(b_{d}\right)$ related to $\rho^{4}$. In particular, the numerator involves the LD between a pair of markers and the denominator the LD between a pair of markers.

Of note, there are two kinds of F2 populations, the conventional F2 that is derived from F1 but not completely reproducible in term of genotypes, and in contrast there is "immortalized F2" (IF2), which can be reproduced accordingly. The IF2 can often be realized in two ways: via double haploid population (DH) (Liu et al. 2017) and from recombination inbred lines (RIL) (Hua et al. 2003). The LD differs upon F2/IF2 is used in practice. Between the $k_{1}^{\text {th }}$ and $k_{2}^{\text {th }}$ markers, for a conventional F2 and DH-derived F2 the squared correlation is $\rho_{k_{1}, k_{2}}^{2}=\left(1-2 c_{k_{1}, k_{2}}\right)^{2}$ but $\rho_{k_{1}, k_{2}}^{2}=$ $\left(\frac{1-2 c_{k_{1}, k_{2}}}{1+2 c_{k_{1}, k_{2}}}\right)^{2}$ for RIL-derived F2. For example, given the recombination of 0.1 between a pair of markers, their $\rho^{2}=$ 0.64 for F2 and DH-derived F2 but 0.44 for RIL-derived F2. For dominance-associated terms, $\rho^{4}=0.41$ for F2 and DHderived IF2, and 0.2 for RIL-derived IF2.

For simplicity, we only consider the typical polygenic trait that the QTLs are randomly distributed along the genome, and, under this assumption, $\sigma_{a}^{2}=-\frac{b_{a}}{2}$ and $\sigma_{d}^{2}=-\frac{b_{d}}{2}$, respectively. A computer program that estimates additive and dominance heritability using Haseman-Elston regression is available from authors. 


\section{Best linear prediction (BLP)}

BLP method was used to predict the genotypic value of each line of the candidate population.

$\hat{g}_{2}=\left(\hat{\sigma}_{a}^{2} \Omega_{a 21}+\hat{\sigma}_{d}^{2} \Omega_{d 21}\right) V^{-1} y_{1}$,

in which $\hat{g}_{2}$ is the predicted genotypic values in the candidate population; $y_{1}$ is the phenotypic values in the training population; $\Omega_{a 21}$ and $\Omega_{d 21}$ represent the additive and the dominance genetic relationship matrix between the candidate and the training population respectively; $\hat{\sigma}_{a}^{2}$ and $\hat{\sigma}_{d}^{2}$ represent the estimated additive and dominance variances respectively; the inverse of the $V$ matrix is computed using $V^{-1}=\left(\hat{\sigma}_{a}^{2} \Omega_{a 11}+\hat{\sigma}_{d}^{2} \Omega_{d 11}+\hat{\sigma}_{e}^{2} I\right)^{-1}$, in which $\Omega_{a 11}$ and $\Omega_{d 11}$ represent the additive and the dominance genetic relationship matrix for the training population respectively.

\section{Results}

\section{Estimates of the heritability and predictability in the simulated $F 2$ population}

We simulated a quantitative trait from F2 experimental population. In the simulated $\mathrm{F} 2$ population, we assumed that 1001 equal-frequent biallelic markers were evenly distributed in one chromosome [the recombination rate was $c$ between the $i$ th and the $(i+1)$ th markers]. All markers were defined as QTLs whose additive and dominance effects follow a normal distribution. Each simulation scenario included 20 replications.

In order to assess the unbiasedness of estimating heritability via the three methods (HEIA, HEIAD, and REMLI AD), we performed a Monte Carlo simulation experiment for a F2 population. When the simulated parameters were set as population size $(n=500)$, additive heritability ( $\left.h_{a}^{2}=0.3\right)$, dominance heritability $\left(h_{d}^{2}=0.2\right)$, and recombination rate $(c=0.01)$, the results showed that $\hat{h}_{a}^{2}=$ $0.271 \pm 0.075$ (via HEIA), $\hat{h}_{a}^{2}=0.271 \pm 0.075$ and $\hat{h}_{d}^{2}=$ $0.193 \pm 0.039$ (via HEIAD), and $\hat{h}_{a}^{2}=0.296 \pm 0.048$ and $\hat{h}_{d}^{2}=0.226 \pm 0.052$ (via REMLIAD) (Fig. 1). It indicated that all three methods could obtain unbiased estimates of parameters under the typical polygenic model.

Moreover, we evaluated the prediction accuracy of HEBLPIAD, HEBLPIA, and GBLUPIAD under five environments in the simulated F2 population (Fig. 2). The size of both the training $\left(n_{T}\right)$ and the candidate population $\left(n_{C}\right)$ were 500 and 100 in all simulations. The squared correlation coefficient $\left(r^{2}\right)$ between the phenotypes and the predicted genotypic values was defined as the prediction accuracy.

In scenario $1\left(h_{a}^{2}=0.4, h_{d}^{2}=0\right.$, and $\left.c=0.01\right)$, the prediction accuracies were HEBLPIAD $=0.333 \pm 0.066$,
GBLUP|AD $=0.314 \pm 0.096, \quad$ and HEBLP|A $=0.335 \pm$ 0.067. In scenario $2\left(h_{a}^{2}=0.40, h_{d}^{2}=0.05\right.$, and $\left.c=0.01\right)$, the prediction accuracies were HEBLP|AD $=0.351 \pm 0.065$, GBLUP|AD $=0.355 \pm 0.066$, and HEBLP|A $=0.334 \pm$ 0.065 . The results of these two simulations indicated that the three methods had a similar predictive ability in the case of no or very small contribution of dominance effects to genetic variation. In scenario $3\left(h_{a}^{2}=0.40, h_{d}^{2}=0.1\right.$, and $c$ $=0.01$ ), the prediction accuracies were HEBLPIAD $=$ $0.388 \pm 0.065$, GBLUP|AD $=0.391 \pm 0.066$, and HEBLP $\mid$ $\mathrm{AD}=0.334 \pm 0.067$. In scenario $4\left(h_{a}^{2}=0.4, h_{d}^{2}=0.2\right.$, and $c=0.01$ ), the prediction accuracies were HEBLP|AD $=$

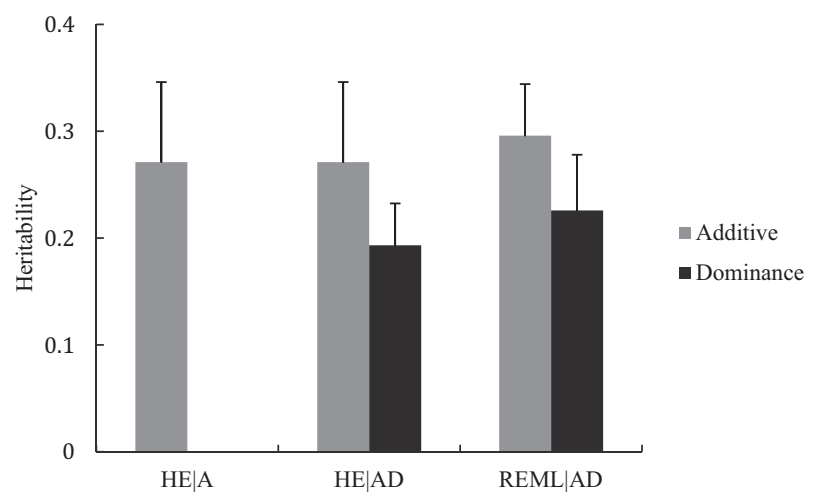

Fig. 1 The estimated heritability of additive and dominance based on a fixed population size (500) via HEIA, HEIAD, and REMLIAD in 20 simulations when additive and dominance heritability was set at 0.3 and 0.2 , respectively, and recombination rate $(c)$ was set as 0.01 . Here the HElA only was used to estimate additive heritability. The vertical bar represents the standard deviation for 20 simulations

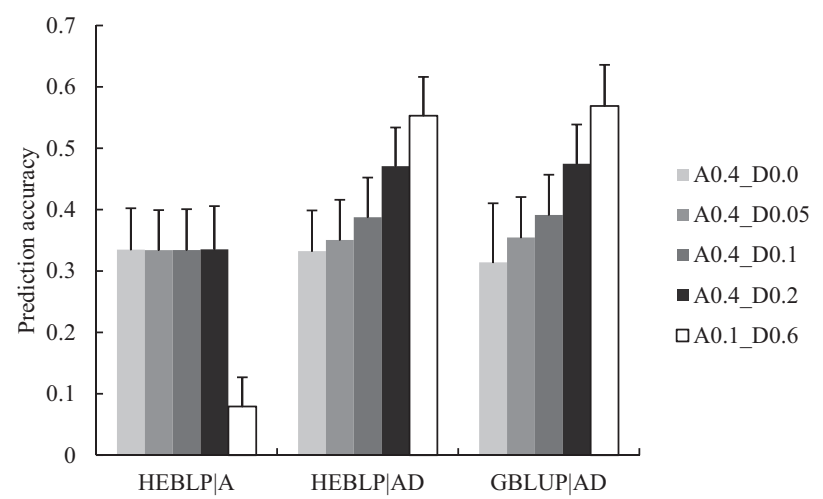

Fig. 2 Predictive ability based on a training population with a fixed population size (500), a candidate population with a fixed sample size (100), and a fixed recombination rate $(c=0.01)$ using HEBLPIA, HEBLPIAD, GBLUPIAD methods in 20 simulations. The value after capital letter A represents additive heritability and that after capital letter D represents dominance heritability (for example, A0.4_D0.0 represents $h_{a}^{2}=0.4$ and $h_{d}^{2}=0.0$ ). The squared correlation coefficient $\left(r^{2}\right)$ between the phenotypes and the predicted genotypic values were defined as the prediction accuracy. The vertical bar represents the standard deviation for 20 simulations 
$0.471 \pm 0.063$, GBLUP|AD $=0.475 \pm 0.064$, and HEBLP|A $=0.335 \pm 0.070$. In scenario $5\left(h_{a}^{2}=0.1, h_{d}^{2}=0.6\right.$, and $c=$ 0.01 ), the prediction accuracies were HEBLPIAD $=0.553$ \pm 0.063 , GBLUPIAD $=0.569 \pm 0.067$, and HEBLP|A $=$ $0.079 \pm 0.048$. It indicated a similar predictability between HEBLPIAD and GBLUPIAD, and a significantly better performance than HEBLPIA in the case of a large contribution of dominance effects to genetic variation.

\section{Comparison of computational time of HE|AD and REML|AD}

We simulated F2 population based on 20 replications to evaluate the computational time of HEIAD and REMLIAD. In this case, the parameters were set as population size $(n=$ $500)$, additive heritability $\left(h_{a}^{2}=0.2\right)$, dominance heritability $\left(h_{d}^{2}=0.6\right)$, marker number $(M=3001)$, and recombination rate $(c=0.01)$. The result showed that $\hat{h}_{a}^{2}=0.183 \pm 0.064$ and $\hat{h}_{d}^{2}=0.568 \pm 0.068$ (via HEIAD), and $\hat{h}_{a}^{2}=$ $0.20 \pm 0.032$ and $\hat{h}_{d}^{2}=0.636 \pm 0.084$ (via REMLIAD), and that HEIAD and REMLIAD took an average of $304 \mathrm{~s}$ and $3487 \mathrm{~s}$ in each simulation, respectively, demonstrating a significant computational advantage of HEIAD over REMLI AD.

\section{Comparison of heritability and predictability between F2 and IF2 derived from RIL using HEBLP| AD}

We simulated F2 and IF2 derived from RIL population to evaluate HEBLPIAD. In this case, we simulated 1001 markers, among which 100 markers were sampled as QTLs. When we estimated the heritability and prediction accuracy, the markers representing QTLs were excluded. Each simulation scenario included 20 replications.

When the simulated parameters were set as training population size $\left(n_{T}=500\right)$, candidate population size $\left(n_{C}=\right.$ $100)$, additive heritability $\left(h_{a}^{2}=0.5\right)$, dominance heritability $\left(h_{d}^{2}=0.25\right)$, and recombination rate $(c=0.01)$, the results of the simulated F2 population showed $\hat{h}_{a}^{2}=0.458 \pm 0.170$, $\hat{h}_{d}^{2}=0.244 \pm 0.111$, and the predictability $r^{2}=0.614 \pm$ 0.061 in the simulated F2 population; for the simulated IF2 populations, $\hat{h}_{a}^{2}=0.480 \pm 0.129, \hat{h}_{d}^{2}=0.230 \pm 0.075$, and the predictability $r^{2}=0.544 \pm 0.071$ in the simulated IF2 population derived from RIL population. As RIL-derived IF2 undergoing multi-generation selfing, its decayed LD resulted a much lower $r^{2}$ than that of F2.

\section{Approximation of prediction accuracy}

To further understand the study, in the Appendix, we derived a formula of prediction accuracy including additive and dominance variance components. This derived result could be considered as an extension to those previously established by Daetwyler et al. (2008) and Goddard (2009).

$r^{2}=H^{2} \frac{H^{2}}{H^{2}+\frac{m_{e \cdot a}+m_{e . d}}{n_{T}}}$.

The result showed that $H^{2}$ was the upper bound of the prediction accuracy, and was further upon (1) the broad heritability $\left(H^{2}=h_{a}^{2}+h_{d}^{2}\right)$, (2) the effective number of markers $\left(m_{e . a}\right)$, (3) the effective number of markers of dominance heritability $\left(m_{e . d}\right)$, and (4) the sample size of the training data. As $m_{e . a}$ and $m_{e . d}$ were determined by the recombination, when the markers were dense, the prediction accuracy could be further approximated as

$r^{2} \approx H^{2} \frac{H^{2}}{H^{2}+\frac{6 l}{n_{T}}}$,

in which $l$ is the length of the chromosome (Morgan). Both Eq 4 and Eq 5 indicated that the upper bound of prediction accuracy was $H^{2}$ when the sample size $n_{T}$ became infinite. Having evaluated the utility of the approximation, we found that the expected and observed prediction accuracy was consistent via HEBLPIAD under different recombination rates based on 10 simulations for F2 population (Table 1). Eq 4 and Eq 5 gave similar prediction for $r^{2}$ when the markers were dense, and the accuracy of Eq 5 was reduced when the markers were sparse. The sample size of the candidate population could only influence the statistical power of the prediction accuracy because $r^{2}$ followed $\chi_{1}^{2}$ under the null hypotheses.

\section{Genomic prediction of 7 traits in the Arabidopsis thaliana $F 2$ population}

The 7 traits, including DTF1, DTF2, DTF3, RLN, CLN, TLN, and LIR1 from a Arabidopsis thaliana F2 population were used to assess the prediction performance of HEBLP| A, HEBLPIAD, and GBLUPIAD.

We first analyzed the 7 traits via HEIA, HEIAD, and REMLIAD, obtaining the estimated additive heritability varying from 0.080 to 0.582 (HEIA), 0.080 to 0.582 (HEl $\mathrm{AD}$ ), and 0.158 to 0.731 (REMLIAD), and the estimated dominance heritability varying from 0.009 to 0.052 (HEl $\mathrm{AD}$ ), and 0.018 to 0.106 (REMLIAD). The results demonstrated that dominance effects only accounted for a little proportion of genetic variation for these traits (Table 2).

Based on 100 replications, we found that the predictability of HEBLPIA, HEBLPIAD, and GBLUPIAD was similar for all traits (Table 3). For example, the prediction accuracies for DTF1 were $0.466 \pm 0.028,0.459 \pm 0.032$, and $0.440 \pm 0.088$ via HEBLPIA, HEBLPIAD, and GBLUPIAD, respectively. It indicated that, as is in the simulations, 
Table 1 Prediction accuracy $\left(r^{2}\right)$ under different recombination rates $(c)$ based on 10 simulations in F2 population when $h_{a}^{2}=0.3$, $h_{d}^{2}=0.5$, marker number $=$ 1001 , and the candidate sample size was 100

\begin{tabular}{lllllllllll}
\hline \multicolumn{9}{c}{$r^{2}$} \\
\multicolumn{10}{c}{$n_{T}=250$} & \multicolumn{3}{c}{$n_{T}=500$} \\
0 & $l$ (Morgan) & $E\left(m_{e . a}\right)^{\mathrm{a}}$ & $E\left(m_{e . a}\right)^{\mathrm{b}}$ & $E\left(r^{2}\right)^{\mathrm{c}}$ & $E\left(r^{2}\right)^{\mathrm{d}}$ & $\hat{r}^{2 \mathrm{e}}$ & $E\left(r^{2}\right)^{\mathrm{c}}$ & $E\left(r^{2}\right)^{\mathrm{d}}$ & $\hat{r}^{2 \mathrm{e}}$ \\
0.05 & 52.68 & 105.57 & 208.34 & 0.312 & 0.39 & $0.309(0.051)$ & 0.448 & 0.448 & $0.497(0.070)$ \\
0.1 & 111.57 & 220.21 & 419.67 & 0.192 & 0.184 & $0.208(0.058)$ & 0.304 & 0.30 & $0.326(0.060)$ \\
0.2 & 255.41 & 471.45 & 771.51 & 0.112 & 0.096 & $0.091(0.046)$ & 0.192 & 0.168 & $0.232(0.084)$ \\
0.3 & 458.15 & 725.10 & 951.08 & 0.088 & 0.054 & $0.078(0.023)$ & 0.152 & 0.104 & $0.183(0.066)$ \\
0.4 & 804.72 & 924.07 & 997.81 & 0.075 & 0.032 & $0.077(0.053)$ & 0.136 & 0.077 & $0.171(0.051)$ \\
0.499 & 3107.30 & 1000.99 & 1001.00 & 0.073 & 0.008 & $0.096(0.044)$ & 0.136 & 0.021 & $0.143(0.062)$ \\
\hline
\end{tabular}

The squared correlation coefficient $\left(r^{2}\right)$ between the phenotypes and the predicted genotypic values was defined as the prediction accuracy.

${ }^{\mathrm{a}} E\left(m_{e, a}\right)$ is calculate from A2. $m_{e . a}=\frac{m^{2}}{m+\sum_{i=1}^{k} \sum_{i \neq j}^{k} e^{-4 d_{i j}}}$, in which $d_{i j}$ is the genetic distance (Morgan) between
marker $i$ and $j$.

${ }^{\mathrm{b}} E\left(m_{e . d}\right)$ is calculated from A3. $m_{e . d}=\frac{m^{2}}{m+\sum_{i=1}^{k} \sum_{i \neq j}^{k} e^{-8 d_{i j}}}$.

${ }^{\mathrm{c}}$ The expected $r^{2}$ was calculated using Eq 4 (or A1) that $r^{2}=H^{2} \frac{H^{2}}{H^{2}+\frac{m_{e . a+m_{e . d}}}{n_{T}}}$. ${ }^{\mathrm{d}}$ The expected $r^{2}$ was calculated using Eq 5 (or A4) that $r^{2}=H^{2} \frac{H^{2}}{H^{2}+\frac{6 \mathrm{I}}{n_{T}}}$, a further approximation when
markers were dense.

$\mathrm{e} \hat{r}^{2}$ represents the mean of the observed values based on 10 simulations and the values in parentheses represent the corresponding standard deviation.
Table 2 The estimated variance proportion $\left(\hat{h}_{a}^{2}\right.$ and $\left.\hat{h}_{d}^{2}\right)$ for the 7 traits in the Arabidopsis thaliana F2 (P19) population

\begin{tabular}{llllll}
\hline Trait & HEIA & HEIAD & & \multicolumn{2}{l}{ REMLIAD } \\
& $\hat{h}_{a}^{2}$ & $\hat{h}_{a}^{2}$ & $\hat{h}_{d}^{2}$ & $\hat{h}_{a}^{2}$ & $\hat{h}_{d}^{2}$ \\
DTF1 & 0.511 & 0.511 & 0.020 & 0.603 & 0.018 \\
DTF2 & 0.403 & 0.402 & 0.025 & 0.544 & 0.022 \\
DTF3 & 0.582 & 0.582 & 0.009 & 0.524 & 0.023 \\
RLN & 0.411 & 0.411 & 0.048 & 0.731 & 0.106 \\
CLN & 0.450 & 0.449 & 0.047 & 0.378 & 0.045 \\
TLN & 0.473 & 0.473 & 0.052 & 0.676 & 0.088 \\
LIR1 & 0.080 & 0.080 & 0.036 & 0.158 & 0.040 \\
\hline
\end{tabular}

HEBLPIA, HEBLPIAD, and GBLUPIAD showed similar predictability in the case of a very small contribution of dominance effects to the genetic variation.

\section{Discussion}

\section{The impact of the dominance heritability on predictive accuracy}

The wide utilization of heterosis in the animals and plants, such as maize, rice, and cattle has significantly increased their productivity. In this study, we extended our previous method of HEBLPIA to HEBLPIAD. The simulation results demonstrated that (1) HEBLPIAD and GBLUPIAD are superior to HEBLPIA when the dominance effects can explain a significant proportion of genetic variation; (2)
HEBLPIAD, GBLUPIAD, and HEBLPIA have a similar predictive ability when the dominance effects can only explain a small proportion of genetic variation. Furthermore, the real data from Arabidopsis thaliana $\mathrm{F} 2$ population was used to evaluate the three methods, and since the estimated heritability showed a small contribution of the dominance effects to genetic variation, the result was supportive to the second case in the simulation. de Almeida Filho et al. (2016) indicated that when the dominance effects consisted of only a small proportion in the total genetic variation, incorporating them into BayesA, BayesB, $\mathrm{BL}$, and BRR would decrease the prediction accuracy. However, it is safe and stable to include dominance effects into HEBLP model under this circumstance. In addition, not limited to the $\mathrm{F} 2$ population as was demonstrated, HEBLPI $\mathrm{AD}$ is applicable as long as the populations promise the estimation of additive and dominance variance components (such as natural population of random mating).

In addition, we also provided an approximation of prediction accuracy for F2 population (Appendix). The genetic length of the chromosome, the density of markers, $H^{2}$, and the sample size of the training population were key factors that would influence the prediction accuracy. The method presented in Appendix was general and could be applied to other populations. In this simulation, we simulated extremely long and single chromosome, which was unrealistic, and we will consider incorporating the real marker density into further study. We considered typical polygenic model only at present, but the interplay between genetic architecture will be included in our further studies. 
Table 3 Prediction accuracy of the 7 traits in the Arabidopsis thaliana F2 (P19) population based on 100 simulations

\begin{tabular}{llllll}
\hline Trait & Training & Candidate & HEBLPIA & HEBLPIAD & GBLUPIAD \\
DTF1 & 100 & 281 & $0.466(0.028)$ & $0.459(0.032)$ & $0.440(0.088)$ \\
DTF2 & 100 & 281 & $0.363(0.030)$ & $0.356(0.032)$ & $0.342(0.071)$ \\
DTF3 & 100 & 277 & $0.501(0.031)$ & $0.494(0.036)$ & $0.485(0.067)$ \\
RLN & 100 & 277 & $0.397(0.033)$ & $0.403(0.034)$ & $0.393(0.069)$ \\
CLN & 100 & 277 & $0.335(0.036)$ & $0.331(0.038)$ & $0.324(0.062)$ \\
TLN & 100 & 277 & $0.437(0.033)$ & $0.444(0.034)$ & $0.429(0.078)$ \\
LIR1 & 100 & 277 & $0.037(0.022)$ & $0.033(0.021)$ & $0.032(0.022)$ \\
\hline
\end{tabular}

The values in parentheses represent standard deviation. The squared correlation coefficient $\left(r^{2}\right)$ between the phenotypes and the predicted genotypic values was defined as the prediction accuracy.

\section{Application of the genomic prediction in hybrid breeding of crops}

The traditional strategy to cultivate hybrid crosses is to perform a large number of cross experiments between the inbred lines and furthermore select desirable hybrids. This process can be accelerated via combining genomic prediction approaches with immortalized F2 (IF2) population constructed by the doubled haploid (DH) population. Hua et al. (2003) first constructed IF2 population, which had the same genetic architecture as the conventional F2 population, can be generated via randomly permutated intermating of recombinant inbred lines (RILs) or DH population at present. In a hybrid breeding program, when sample size $(n)$ of RIL or DH population is large and all crosses $\left[\frac{n(n-1)}{2}\right]$ between inbred lines from the RIL or DH population need to be evaluated in the field trials, it will occupy large resources. To reduce the cost of genetic improvement, genomic prediction can be used to IF2 population to select hybrid crosses with high-hybrid performance. Guo et al. (2013) applied genomic prediction to an IF2 population derived from RIL population in maize, and Xu et al. (2014) did that in rice. Liu et al. (2017) has applied genomic prediction to IF2 population based on rapeseed $\mathrm{DH}$ population. However, construction of RIL population is time-consuming, and therefore the procedure of GP $+\mathrm{IF} 2$ (DH) will be a more efficient choice to pick out superior hybrids and potential lines with high-specific combining ability or general combining ability.

Acknowledgements This study was supported by the National Natural Science Foundation of China (31771392 to G.-B.C.).

Author contributions H.L. and G.-B.C. designed and performed the study as well as wrote the manuscript.

\section{Compliance with ethical standards}

Conflict of interest The authors declare that they have no conflict of interest.

\section{Appendix}

\section{Factors influence prediction accuracy for F2 population}

In this note, we try to outline the factors that influence the prediction accuracy for an F2 population.

For the training population, its phenotype can be expressed as

$y=\mu+\sum_{j=1}^{m} b_{a_{j}} x_{a_{j}}+\sum_{j=1}^{m} b_{d_{j}} x_{d_{j}}+\varepsilon$.

Here, we assume every marker is causal and has small effects, a typical polygenic trait. $x_{a_{j}}$ and $x_{d_{j}}$ are the orthogonal coding of the $j$ th marker for the additive and dominance effect. $\operatorname{var}(y)=V_{p}$ the phenotypic variance, and $\operatorname{var}\left(\sum_{j=1}^{m} b_{a_{j}} x_{a_{j}}+\sum_{j=1}^{m} b_{d_{j}} x_{d_{j}}\right)=h_{A}^{2}+h_{D}^{2}=H^{2}$.

According to linear regression theory, for the additive effect for the $j$ th marker can be estimated as $\hat{b}_{a_{j}}=\frac{\operatorname{cov}\left(y, x_{a_{j}}\right)}{\operatorname{var}\left(x_{a_{j}}\right)}$ and rewritten as $b_{a_{j}}+\sigma_{\hat{b}_{a_{j}}}$, in which $\sigma_{\hat{b}_{a_{j}}}=\frac{\sigma_{e}^{2}}{N_{T} \sigma_{x_{j}}^{2}}$ the sampling variance of the estimate; for the dominance effect, $\hat{b}_{d_{j}}=\frac{\operatorname{cov}\left(y, x_{d_{j}}\right)}{\operatorname{var}\left(x_{d_{j}}\right)}$, and $\sigma_{\hat{b}_{d_{j}}}=\frac{\sigma_{e}^{2}}{N_{T} \sigma_{x_{d_{j}}}^{2}} . N_{T}$ is the sample size of the training population, and $m$ is the number of markers.

For the candidate population, the phenotype can be expressed as $\quad y_{C}=a+\sum_{j=1}^{k} b_{a_{j}} \tilde{x}_{a_{j}}+\sum_{j=1}^{k} b_{d_{j}} \tilde{x}_{d_{j}}+\varepsilon_{T}$, while the predicted genotypic values $\hat{y}_{C}=\sum_{j=1}^{k} \hat{b}_{a_{j}} \tilde{x}_{a_{j}}+\sum_{j=1}^{k} \hat{b}_{d_{j}} \tilde{x}_{d_{j}}$. It is easy to derive the variance and covariance terms below.

$\operatorname{Var}\left(y_{C}\right)=\sum_{j=1}^{k} b_{a_{j}}^{2} \sigma_{x_{a_{j}}}^{2}+\sum_{j=1}^{k} b_{d_{j}}^{2} \sigma_{x_{d_{j}}}^{2}+\sigma_{e_{T}}^{2}=V_{G}+V_{e_{C}}$, 


$$
\begin{array}{r}
\operatorname{Var}\left(\hat{y}_{C}\right)=\sum_{j=1}^{k} b_{a_{j}}^{2} \sigma_{x_{a_{j}}}^{2}+\sum_{j=1}^{k} b_{d_{j}}^{2} \sigma_{x_{d_{j}}}^{2}+\left(\frac{m_{e . a}}{n_{T}}+\frac{m_{e . d}}{n_{T}}\right) \sigma_{e}^{2} \\
=V_{G}+V_{e}\left(\frac{m_{e . a}}{n_{T}}+\frac{m_{e . d}}{n_{T}}\right)
\end{array}
$$

$\operatorname{Cov}\left(\hat{y}_{T}, y_{T}\right)=\sum_{j=1}^{k} b_{a_{j}}^{2} \sigma_{x_{a_{j}}}^{2}+\sum_{j=1}^{k} b_{d_{j}}^{2} \sigma_{x_{d_{j}}}^{2}=V_{G}$

The prediction accuracy is

$$
\begin{aligned}
& r^{2}=\frac{\operatorname{Cov}\left(\hat{y}_{T}, y_{T}\right)^{2}}{\operatorname{Var}\left(y_{T}\right) \operatorname{Var}\left(\hat{y}_{T}\right)}=\frac{V_{G}^{2}}{\left(V_{G}+V_{e_{T}}\right)\left[V_{G}+V_{e}\left(\frac{m_{e . a}}{n_{T}}+\frac{m_{e . d}}{n_{T}}\right)\right]} \\
& =H^{2} \frac{H^{2}}{\left[H^{2}+\left(1-H^{2}\right)\left(\frac{m_{e . a}}{n_{T}}+\frac{m_{e . d}}{n_{T}}\right)\right]} \approx H^{2} \frac{H^{2}}{H^{2}+\frac{m_{e \cdot a}+m_{e . d}}{n_{T}}}
\end{aligned} .
$$

For genetic value

$y_{G}=\mu+\sum_{j=1}^{k} b_{a_{j}} x_{a_{j}}+\sum_{j=1}^{k} b_{d_{j}} x_{d_{j}}$

$V\left(y_{G}\right)=V_{G}$

The prediction accuracy between the true genotypic values and the predicted genotypic values can be written as squared Pearson's correlation

$r_{G}^{2}=\frac{V_{G}^{2}}{V_{G}\left[V_{G}+V_{e} \frac{m_{e . a}+m_{e . d}}{n_{T}}\right]}=\frac{H^{2}}{H^{2}+\frac{m_{e . a}+m_{e . d}}{n_{T}}}$.

This equation is an extension of the one as derived by Daetwyler et al. (2008), but here we include the dominance component. In practice, the prediction accuracy is more relevant to the effective number of loci, which can be understood as quasi-independent segment of the whole genome. So, the prediction accuracy is approximated as

$r^{2}=H^{2} \frac{H^{2}}{H^{2}+\frac{m_{e . a}+m_{e . d}}{n_{T}}}=H^{2} r_{G}^{2}$

in which $m_{e, a}$ and $m_{e, d}$ are the effective number of markers coded for additive and dominance effects.

$m_{e . a}=\frac{m^{2}}{m+\sum_{i=1}^{k} \sum_{i \neq j}^{k} r_{i j}^{2}}$
As for markers not on the same chromosome, the LD is nearly zero, so $m_{e . a}=\frac{m^{2}}{m+\sum_{i=1}^{k} \sum_{i \neq j}^{k} r_{i j}^{2}}$

$$
m_{e . d}=\frac{m^{2}}{m+\sum_{i=1}^{k} \sum_{i \neq j}^{k} r_{i j}^{4}} \text {. }
$$

If the recombination is based on Haldane map function, for $\mathrm{F} 2 r_{i j}^{2}=\exp \left(-4\left|d_{i}-d_{j}\right|\right)=e^{-4 d_{i j}}$, in which $d_{i, j}=\mid d_{i}$ $-d_{j}$ l is the genetic distance (Morgan) between a pair of loci, and $r_{i j}^{2}=e^{-8 d_{i j}}$. Obviously, when there is no LD between markers, $r_{i j}^{2}=0$, and $m_{e, a}=m, m_{e, d}=m$. As $r_{i j}^{4} \leq r_{i j}^{2}$, we have $m_{e . a} \leq m_{e . d} \leq m$.

\section{Further approximation for the prediction accuracy}

For the additive component,

$$
\frac{\sum_{i=1}^{k} \sum_{i \neq j}^{k} r_{i j}^{2}}{m^{2}}={ }_{000}^{l} l e^{-4\left|d_{x_{1}}-d_{x_{2}}\right|} d_{x_{1}} d_{x_{2}}=\frac{1}{2 l^{2}}\left(l-\frac{c_{2 l}}{2}\right)
$$

and for the dominance component,

$$
\frac{\sum_{i=1}^{k} \sum_{i \neq j}^{k} r_{i j}^{4}}{m^{2}}={ }_{00}^{l l} l e^{-4\left|d_{x_{1}}-d_{x_{2}}\right|} d_{x_{1}} d_{x_{2}}=\frac{1}{4 l^{2}}\left(l-\frac{c_{2 l}}{2}\right)
$$

in which $c_{2 l}$ is the recombination fraction given the genetic

distance of $2 l$.
So, $m_{e . a}=\left[\frac{1}{m}+\frac{\left(l_{1}-\frac{c_{2 l} l}{2}\right)}{2 l^{2}}\right]^{-1}$, if the markers are dense, and $m>>l_{1}$ ( $m$ is often greater than 10,000 along a single chromosome), $m_{e . a} \approx 2 l$; similarly, $m_{e . a} \approx 4 l$. So, the prediction accuracy can be further approximated as

$$
r^{2} \approx H^{2} \frac{H^{2}}{H^{2}+\frac{6 l}{n_{T}}}
$$

when the density of markers is high.

So the expectation of the prediction accuracy is upon the training sample size, but the statistical significance of $r^{2}$ depends on the sample size of the candidate sample size. Under the null distribution $r^{2}$ follows $\chi_{1}^{2}$, so the noncentrality parameter for the statistical test of $r^{2}$ is $\lambda=\frac{n_{C} r^{2}}{1-r^{2}}$, in which $n_{C}$ is the sample size of the candidate population.

In genomic prediction, the additive genomic relationship matrix can be used to estimate $m_{e . a}$. Given $A$, an $n_{T} \times n_{T}$ matrix, the additive genomic relationship matrix, if we estimate variance, $\sigma_{A_{o}}^{2}$, of the $\frac{n_{T}\left(n_{T}-1\right)}{2}$ off-diagonal elements, and $\hat{m}_{e . a}=\frac{1}{\sigma_{A_{o}}^{2}}$; similarly, we can have $\hat{m}_{e . d}=\frac{1}{\sigma_{D_{o}}^{2}}$ for the dominance effective number of markers. 


\section{References}

Arruda MP, Lipka AE, Brown PJ, Krill AM, Thurber C, BrownGuedira G et al. (2016) Comparing genomic selection and marker-assisted selection for Fusarium head blight resistance in wheat (Triticum aestivum L.). Mol Breed 36:84

Bernardo R, Yu J (2007) Prospects for genomewide selection for quantitative traits in maize. Crop Sci 47:1082-1090

Calus MPL, Meuwissen THE, De Roos APW, Veerkamp RF (2008) Accuracy of genomic selection using different methods to define haplotypes. Genetics 178:553-561

Chen G-B (2014) Estimating heritability of complex traits from genome-wide association studies using IBS-based HasemanElston regression. Front Genet 5:107

Daetwyler HD, Villanueva B, Woolliams JA (2008) Accuracy of predicting the genetic risk of disease using a genome-wide approach. PLoS ONE 3:e3395

de Almeida Filho JE, Guimarães JFR, e Silva FF, de Resende MDV, Muñoz P, Kirst M et al. (2016) The contribution of dominance to phenotype prediction in a pine breeding and simulated population. Heredity 117:33-41

Denis M, Bouvet J-M (2011) Genomic selection in tree breeding: testing accuracy of prediction models including dominance effect. BMC Proc 5:013

Denis M, Bouvet JM (2013) Efficiency of genomic selection with models including dominance effect in the context of Eucalyptus breeding. Tree Genet Genomes 9:37-51

Goddard M (2009) Genomic selection: prediction of accuracy and maximisation of long term reponse. Genetica 136:245-257

Guo T, Li H, Yan J, Tang J, Li J, Zhang Z et al. (2013) Performance prediction of $\mathrm{F} 1$ hybrids between recombinant inbred lines derived from two elite maize inbred lines. Theor Appl Genet 126:189-201

Hayes BJ, Bowman PJ, Chamberlain AJ, Goddard ME (2009) Genomic selection in dairy cattle: progress and challenges. $\mathrm{J}$ Dairy Sci 92:433-443

Heffner EL, Sorrells ME, Jannink JL (2009) Genomic selection for crop improvement. Crop Sci 49:1-12

Hill WG, Robertson A (1968) Linkage disequilibrium in finite populations. Theor Appl Genet 38:226-231

Hua J, Xing Y, Wu W, Xu C, Sun X, Yu S et al. (2003) Single-locus heterotic effects and dominance by dominance interactions can adequately explain the genetic basis of heterosis in an elite rice hybrid. Proc Natl Acad Sci USA 100:2574-2579

Jannink J-L, Lorenz AJ, Iwata H (2010) Genomic selection in plant breeding: from theory to practice. Brief Funct Genom 9:166-177

Li L, Lu K, Chen Z, Mu T, Hu Z, Li X (2008) Dominance, overdominance and epistasis condition the heterosis in two heterotic rice hybrids. Genetics 180:1725-1742
Liu H, Chen G-B (2017) A fast genomic selection approach for large genomic data. Theor Appl Genet 130:1277-1284

Liu P, Zhao Y, Liu G, Wang M, Hu D, Hu J et al. (2017) Hybrid performance of an immortalized $\mathrm{F} 2$ rapeseed population is driven by additive, dominance, and epistatic effects. Front Plant Sci 8:815

Meuwissen TH, Hayes BJ, Goddard ME (2001) Prediction of total genetic value using genome-wide dense marker maps. Genetics 157:1819-1829

Nishio M, Satoh M (2014) Including dominance effects in the genomic BLUP method for genomic evaluation. PLoS ONE 9:e85792

Resende RT, Resende MDV, Silva FF, Azevedo CF, Takahashi EK, Silva-Junior OB et al. (2017) Assessing the expected response to genomic selection of individuals and families in Eucalyptus breeding with an additive-dominant model. Heredity 119:245-255

Riedelsheimer C, Czedik-Eysenberg A, Grieder C, Lisec J, Technow F, Sulpice R et al. (2012) Genomic and metabolic prediction of complex heterotic traits in hybrid maize. Nat Genet 44:217-220

Salomé PA, Bomblies K, Laitinen RAE, Yant L, Mott R, Weigel D (2011) Genetic architecture of flowering-time variation in Arabidopsis thaliana. Genetics 188:421-433

Schaeffer LR (2006) Strategy for applying genome wide selection in dairy cattle. J Anim Breed Genet 123:218-223

Su G, Christensen OF, Ostersen T, Henryon M, Lund MS (2012) Estimating additive and non-additive genetic variances and predicting genetic merits using genome-wide dense single nucleotide polymorphism markers. PLoS ONE 7:e45293

Technow F, Riedelsheimer C, Schrag TA, Melchinger AE (2012) Genomic prediction of hybrid performance in maize with models incorporating dominance and population specific marker effects. Theor Appl Genet 125:1181-1194

VanRaden PM (2008) Efficient methods to compute genomic predictions. J Dairy Sci 91:4414-4423

Vitezica ZG, Legarra A, Toro MA, Varona L (2017) Orthogonal estimates of variances for additive, dominance, and epistatic effects in populations. Genetics 206:1297-1307

Wang X, Li L, Yang Z, Zheng X, Yu S, Xu C et al. (2017) Predicting rice hybrid performance using univariate and multivariate GBLUP models based on North Carolina mating design II. Heredity 118:302-310

Xu S, Zhu D, Zhang Q (2014) Predicting hybrid performance in rice using genomic best linear unbiased prediction. Proc Natl Acad Sci USA 111:12456-12461

Zhang Z, Zhang Q, Ding XD (2011) Advances in genomic selection in domestic animals. Chin Sci Bull 56:2655-2663 